Gut, 1969, 10, 681-683

\title{
$X$-ray diffraction studies of the composition of gallstones from English and Australian patients
}

\author{
D. JUNE SUTOR AND SUSAN E. WOOLEY \\ From the Department of Chemistry, University College, London
}

SUMMARY The crystalline composition of two collections of gallstones from patients in England and Australia have been determined by the $x$-ray powder diffraction technique. Twelve substances have been identified including a form of cholesterol which hitherto has not been reported. The weighted percentage composition averaged over each collection shows that cholesterol is the major constituent of the Australian stones and cholesterol and cholesterol monohydrate the major constituents of the English calculi. The cholesterol is possibly a decomposition product of the monohydrate. The calcium carbonates-calcite, aragonite, and vaterite-constitute most of the remainder of the calculi. Although their percentage composition is much smaller than that of the cholesterols, they are nevertheless present in a high proportion of stones. Small traces of apatite, whitlockite, sodium chloride, calcium stearate and palmitic acid (or other long-chain compounds having closely related spacings) have been found. Small spheroids scattered throughout some stones appear to be mainly calcium stearate, although the total quantity available is too small and too impure for a definite identification.

Only a few people have studied the composition of biliary calculi (see references in Bogren, 1964). Their work has been mainly concerned with the identification of the different substances present and the methods used have been chemical analysis, infrared spectroscopy, and $x$-ray diffraction. The $x$-ray diffraction technique has definite advantages over chemical analysis. Chemical methods determine the presence of groups and elements but cannot always identify a compound as such. Crystallographic methods can usually identify rapidly and certainly crystalline samples of pure compounds or components of mixtures provided that a minimum quantity is present. Minor components may sometimes, however, be missed (Sutor, 1968). We are using the $x$-ray powder method in an investigation into the composition of the crystalline material in the gallstones of patients from different countries and when enough information becomes available the data will be correlated with any relevant factors such as diet, age, and occupation.

\section{METHOD}

The $x$-ray powder method is a rapid, non-destructive technique for the identification of crystalline substances. Amorphous material cannot be identified by this method. When a sample (say $0.1 \mathrm{mg}$ ) of a crystalline powder is rotated in a pencil (say $\frac{1}{2} \mathrm{~mm}$ diameter) of monochromatic $x$ rays, the resulting diffraction pattern recorded on photographic film placed a few centimetres away consists of many fine concentric rings (or lines if only part of the rings is recorded) whose intensities and positions on the film are related to the atomic arrangement in the crystal. These quantities are therefore characteristic of a particular crystalline substance and the first three or four lines are usually sufficient for identification purposes. The intensities can be measured quantitatively, either with a photometer or by visual comparison with a standard scale, and qualitatively by designating each ring, strong, weak, or medium. From the diameter of any ring on the film, the interplanar spacing $d$ of the set of atomic planes giving rise to that ring can be calculated.

Two experimental methods have been used.

1 When only a small amount of material (linear dimensions about 0.2 to $0.5 \mathrm{~mm}$ ) was available, the specimen was mounted on a glass fibre and rotated in the collimated $x$-ray beam. The diffraction pattern was recorded using a cylindrical camera of radius $3 \mathrm{~cm}$ and $x$-radiation from a copper target (wavelength $1.54 \AA$ ).

2 When more material was available, better resolution of the diffraction pattern for identification purposes was obtained by using a focusing technique such as that employed in the Nonius powder camera, which has a specimen-to-film distance of $5.73 \mathrm{~cm}$ and takes four specimens at a time, in a special holder.

A patient usually forms multiple gallstones but 
frequently only one stone was sent to us, so this work relates to the percentage composition of one calculus per patient irrespective of size and weight.

The stones were only sampled. They were split open with a microtome given a gentle tap with a hammer. Small specimens were then taken from the nucleus (if any), the various interior layers or regions, and the surface. The specimens were carefully powdered and then photographed using filtered $(\mathrm{CuK} \alpha)$ radiation. The resulting diffraction patterns were compared with a chart of standard photographs of known gallstone constituents: L1, anhydrous cholesterol; L2, cholesterol monohydrate; L3, a second form of anhydrous cholesterol or an intermediate in the conversion of the monohydrate to the anhydrous material; M1, calcite; M2, aragonite; M3, vaterite; $N$, calcium stearate; $P$, palmitic acid; $Q$, sodium chloride; F1, carbonate-apatite (only distinguishable from hydroxyapatite by a chemical test for carbon dioxide); F2, hydroxyapatite; and F4, whitlockite. L3, which has not been reported by other workers, is one of the forms obtained when anhydrous cholesterol is allowed to crystallize from benzene or chloroform. Because of contamination by $\mathrm{L} 2$ it has not yet been possible to prepare samples of L3 pure enough for chemical analysis.

Samples consisting of mixtures of several substances can usually be identified but if the strong lines of a minor component are obscured by lines of the major components identification can be more difficult. This masking effect is only likely to occur here with mixtures of vaterite and aragonite.

\section{PRESENTATION OF RESULTS}

The results are presented in two different ways: first according to the percentage number of stones in a given collection which contain an observable amount of a compound, and second according to the weighted percentage of each compound present averaged over all stones in the given collection. The system of weighting was the same as that used in our work on the composition of urinary calculi (Lonsdale, Sutor, and Wooley, 1968). The whole stone was arbitrarily given a weight of 18 which was divided equally among the three regions: nucleus, interior, and surface. If the nucleus and interior appeared homogeneous, a weight of 12 was assigned to their representative sample and 6 to the surface. These weights were then further divided among the various substances present according to the number of substances and the relative intensity of their diffraction patterns.

\section{MATERIAL}

Two collections of stones were examined. The stones from England were from patients aged 20 to 78 years (27 women, 19 men, and one unknown). Those from Australia were from patients aged 41 to 77 years (13 women, seven men and one unknown).

\section{RESULTS}

The results are given in Table 1 and are expressed in terms of the different constituents found in the gallstones.

CHOLESTEROL In both collections the major contituent was cholesterol. When the stone had a sharply defined nucleus this region consisted wholly or partly of this substance. Once cholesterol is laid down in a calculus, subsequent regions almost always contain observable amounts. Bogren and Larsson (1963) have examined cholesterol deposits (including 100 biliary calculi) from man immediately after they were removed at operation or necropsy and found that in all cases the material was cholesterol monohydrate. They also showed that the monohydrate can lose water with the formation of the anhydrous form. With very small crystals the transformation takes place in a few days, but large crystals are stable for up to five years under certain conditions. We have found both forms of cholesterol. The English stones were removed during the past two years but we have not examined any freshly removed stones. The dates of removal of the Australian calculi are not known. Probably the anhydrous cholesterol was formed from the monohydrate but whether this is always the case or whether anhydrous cholesterol is ever deposited as such is not yet known.

We have obtained a third diffraction pattern for cholesterol which may correspond to an intermediate in the decomposition reaction or more likely to another crystalline form of anhydrous cholesterol. The

TABLE I

RESULTS OF EXAMINATION OF COMPONENTS IN ENGLISH AND AUSTRALIAN GALLSTONES

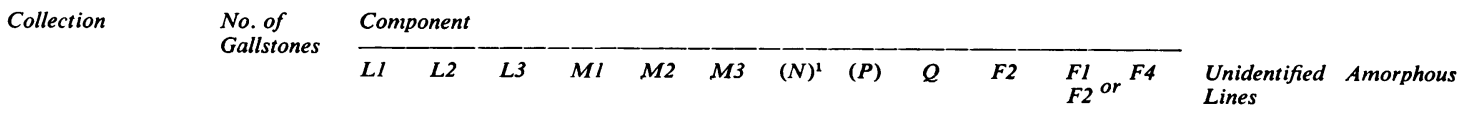

\begin{tabular}{|c|c|c|c|c|c|c|c|c|c|c|c|c|c|c|c|}
\hline \multirow{3}{*}{$\begin{array}{l}\text { English } \\
\text { Australian }\end{array}$} & \multicolumn{15}{|c|}{ Percentage occurrence } \\
\hline & 47 & 50 & 54 & 13 & 35 & 33 & 41 & (15) & (2) & 8 & 2 & 2 & - & 13 & - \\
\hline & 21 & 100 & 10 & 25 & 30 & 5 & 40 & (20) & - & 15 & 5 & - & 5 & 5 & 一 \\
\hline & \multicolumn{15}{|c|}{ Weighted percentage } \\
\hline English & 47 & 26 & 29 & 3 & 8 & 12 & 10 & (2) & (1) & 3 & $>0$ & $>0$ & - & 1 & 5 \\
\hline Australian & 21 & 77 & 1 & 2 & 4 & 1 & 9 & (1) & - & 3 & 1 & - & 1 & 1 & - \\
\hline
\end{tabular}

${ }^{1}$ Brackets indicate uncertainty of identification 
new diffraction pattern resembles closely that of the anhydrous material. Apart from the disappearance of a few weak lines from the new pattern, the only difference is in the position and intensity of four of the strong lines (Table II).

TABLE II

INTERPLANAR SPACING $(d)$ AND INTENSITY OF FOUR OF THE STRONG LINES IN ANHYDROUS CHOLESTEROL AND THE NEW MATERIAL

\begin{tabular}{lllll}
\multicolumn{2}{l}{ Anhydrous } & Cholesterol $(L I)$ & & \multicolumn{2}{l}{ New Material $(L 3)$} \\
\cline { 1 - 1 }$(\AA)$ & Intensity & & $d(\AA)^{1}$ & Intensity \\
\hline $5 \cdot 74$ & Strong & $5 \cdot 6$ & Strong \\
$5 \cdot 23$ & Strong & $5 \cdot 3$ & Strong \\
5.09 & Strong & & $5 \cdot 15$ & Strong \\
4.90 & Strong & & 4.95 & Medium
\end{tabular}

${ }^{1} d$-spacings for the new material may be subject to a small error in origin since no internal standard was used in making the measurements.

CALCIUM CARBONATE The three forms of calcium carbonate-calcite, aragonite, and vaterite-make up most of the remainder of the stones. Calcite has been widely reported elsewhere in nature but aragonite and vaterite are less common forms. Although their percentage composition is not so great as that of the cholesterols, the carbonates nevertheless occur in a high proportion of calculi. Some of the stones consist solely of calcium carbonate and one very unusual stone contained only aragonite. Calcite, aragonite, and vaterite are frequently found with cholesterol, and Lonsdale (1968) has shown that the close epitaxial relationships among these compounds might account for the formation of these mixed calculi.

Calcium STEARaTe Bogren (1964) has stated that stones containing calcium stearate are found in Japan but not in western countries. Calcium stearate (or some other long-chain compound having closely related spacings) appears to be present in about $20 \%$ of the Australian and in $15 \%$ of the English stones. It occurs as small spheroids in calculi which do not show radial striations but consists of aggregates of fairly loosely packed material. Small groups of these spheroids are usually scattered through the stone but form only a very small part of it.

SODIUM CHLORIDE Several calculi contain sodium chloride. The reason for the occurrence of this waterand bile-soluble salt is not known. All other constituents of biliary calculi are relatively insoluble and no water-soluble substances have been found in urinary calculi (Sutor and Scheidt, 1968).

OTHER COMPONENTS The remaining constituents consist of only very small amounts of hydroxyapatite, possibly carbonate-apatite (impossible to distinguish from hydroxyapatite in the presence of the calcium carbonates), whitlockite $\left(\beta-\mathrm{Ca}_{3}\left(\mathrm{PO}_{4}\right)_{2}\right)$, and palmitic acid $\left(\mathrm{C}_{15} \mathrm{H}_{31} \mathrm{COOH}\right)$ or another longchain compound having closely related spacings. Apatite frequently occurs in finely divided form and its $x$-ray pattern is therefore very diffuse, so that its presence is difficult to detect by this method. Two of the stones and the nucleus of another were almost completely amorphous but one of the amorphous stones which had been analysed chemically (Kirk, 1968) consisted of calcium phosphate.

No bile pigments were identified but these too are usually amorphous.

We thank Professor Dame Kathleen Lonsdale for her interest in this work, Professor A. J. Harding Rains, and Mr C. J. C. Kirk for providing the English stones, Professor M. R. Ewing and Dr M. Wheeler for the Australian stones, and the Medical Research Council for financial assistance.

\section{REFERENCES}

Bogren, H. (1964). The composition and structure of Human gallstones. Acta radiol. (Diagm.) (Stockh.) Supplementum, 226.

-, and Larsson, K. (1963). An $x$-ray-diffraction study of crystalline cholesterol in some pathological deposits in man. Biochim. biophys. Acta (Amst), 75, 65-69.

Kirk, C. J. C. (1968). Private communication.

Lonsdale, K. (1968). Epitaxy as a growth factor in urinary calculi and gallstones. Nature, 217, 56-58.

- Sutor, D. J., and Wooley, S. (1968). Composition of urinary calculi by $x$-ray diffraction. Collected data from various localities. I. Norwich (England) and district, 1773-1961. Brit. J. Urol., 40. 33-36.

Sutor, D. J. (1968). Difficulties in the identification of components of mixed urinary calculi using the $x$-ray powder method. Ibid, 40, 29-32.

- and Scheidt, S. (1968). Identification standards for human urinary calculus components, using crystallographic methods. Ibid, 40, 22-28. 